\title{
Arterial Spin-Labeling to Discriminate Pediatric Cervicofacial Soft-Tissue Vascular Anomalies
}

\author{
(D) G. Boulouis, (DV. Dangouloff-Ros, (D) O. Boccara, (D) N. Garabedian, (DV. Soupre, (D)A. Picard, (D) V. Couloigner, (D) N. Boddaert, \\ (D) O. Naggara, and DF. Brunelle
}

\begin{abstract}
BACKGROUND AND PURPOSE: Differentiating major subtypes of cervicofacial vascular lesions is crucial for appropriate management. The aim of our study was to evaluate the performance of an MR imaging arterial spin-labeling perfusion sequence in discriminating pediatric cervicofacial soft-tissue vascular anomalies.

MATERIALS AND METHODS: We conducted a retrospective analysis of data from a prospectively maintained registry including pediatric patients at a tertiary pediatric center between January 2012 and January 2014. We included pediatric patients with a final diagnosis of soft-tissue vascular anomalies and an MR imaging, including an arterial spin-labeling sequence at presentation. We performed an analysis of lesion perfusion, blinded to clinical data, by using concurrent spiral 3D pseudocontinuous arterial spin-labeling (1.5T magnet; spiral matrix, $512 \times 8 \mathrm{~mm}$; postlabeling delay, $1025 \mathrm{~ms}$ ). Lesional flow was recorded with calibrated intralesional ROls. Perfusion characteristics were compared among lesion subtypes with the Mood Median test.

RESULTS: Among 840 patients screened, 46 matched the inclusion criteria and were included (median age, 1.45 years; interquartile range, $0.4-5.1$ years; 27 females). Hemangiomas, including infantile hemangiomas ( $n=18$ patients) and noninvoluting $(n=2)$ and rapidly involuting $(n=1)$ congenital types, demonstrated marked hyperperfusion (median flow, $436 \mathrm{~mL} / \mathrm{min} / 100 \mathrm{~g}$; interquartile range, $212.5-603 \mathrm{~mL} / \mathrm{min} / 100 \mathrm{~g}$ ), significantly higher than that of lymphatic malformations (median, $22.5 \mathrm{~mL} / \mathrm{min} / 100 \mathrm{~g}$; interquartile range, $16-60 \mathrm{~mL} / \mathrm{min} / 100 \mathrm{~g}$; $P<.001$ ) or venous malformations (median, $25 \mathrm{~mL} / \mathrm{min} / 100 \mathrm{~g}$; interquartile range, $15-66.5 \mathrm{~mL} / \mathrm{min} / 100 \mathrm{~g} ; P=.003$ ).
\end{abstract}

CONCLUSIONS: MR imaging arterial spin-labeling is a valuable tool for the assessment of soft-tissue vascular anomaly hemodynamics and for the classification of major lesion subtypes.

ABBREVIATIONS: ASL = arterial spin-labeling; $\mathrm{HI}=$ infantile hemangioma; $\mathrm{IQR}=$ interquartile range; $\mathrm{NICH}=$ noninvoluting congenital hemangioma; $\mathrm{RICH}=$ rapidly involuting congenital hemangioma; STVA = soft-tissue vascular anomaly

S oft-tissue vascular anomalies (STVAs) include vascular tumors and vascular malformations; they can involve any part of the body and can be responsible for non negligible morbidity. ${ }^{1}$ Vascular lesions represent the first cause of soft-tissue tumors and

Received July 20, 2016; accepted after revision October 18.

From the Department of Pediatric Radiology (G.B., V.D.-R., N.B., O.N., F.B.), Institut National de la Santé et de la Recherche Médicale U1000 (V.D.-R., N.B., F.B.), and Institut National de la Santé et de la Recherche Médicale U894 (G.B., O.N.), Descartes University, Paris, France; Departments of Pediatric Dermatology (O.B.), Pediatric Oto-Rhino-Laryngology (O.B., N.G., V.C.), and Pediatric Maxillo-Facial and Plastic Surgery (O.B., V.S., A.P., O.N.), Necker Children's Hospital, Descartes University, Paris, France; Centre de Référence des Malformations Rares de la Face de la Cavite Buccale (O.B., V.S., A.P.), Maxillofacial Malformation Reference Center, Paris,

France; Faculté de Médecine Paris-Descartes (O.B., N.G., V.S., A.P., V.C., N.B., F.B.), Université Paris 5, Paris, France; Department of Neuroradiology (G.B., O.N.), Centre Hospitalier Sainte Anne, Paris, France; and Unite Mixte de Recherche 1163 (N.B.,

F.B.), Institut Imagine, Paris, France.

Please address correspondence to Gregoire Boulouis, MD, MSc, Stroke Research Center, 175 Cambridge St, Suite 300, Boston, MA 02114; e-mail: gregoireboulouis@ gmail.com; @gboulouis

http://dx.doi.org/10.3174/ajnr.A5065 pseudotumors in children. ${ }^{2}$ The International Society for the Study of Vascular Anomalies has classified these lesions in a framework of consistent nomenclature, last updated in April 2014. ${ }^{3}$ This nomenclature is based on a body of clinical, biologic, pathologic, genetic, and imaging data. In this classification, perfusion characteristics play a central role in lesion definition ${ }^{3}$; therefore, any technique allowing local blood perfusion measurement is of critical importance for the diagnostic work-up. ${ }^{4,5}$ In this behavior-based lesion classification, vascular anomalies are divided into vascular tumors, which include hemangiomas (benign tumor subgroup) and vascular malformations, which include lymphatic and venous lesions (locally aggressive or borderline subgroup). Differentiating these major subtypes of vascular lesions is crucial for appropriate management.

The sonographic appearance and flow pattern on Doppler examinations are the most commonly available diagnostic tests to classify STVAs in most centers; however, their use for cervicofacial STVAs can be challenging. Intracranial extensions are obvi- 
ously out of reach when the fontanels are closed, and the extent of the lesions can be underestimated when assessed solely by ultrasound, especially when they are embedded or hidden by bony structures. $^{5}$

MR imaging has emerged as a key complementary examination for these complex or deep vascular lesions ${ }^{2,5,6}$ or when examination of the brain is mandated by potential lesion associations. ${ }^{2,6,7}$ In such situations, contrast medium injection may be required to assess the enhancement pattern of the lesion. ${ }^{8}$ However, besides needing a venous access, contrast enhancement is not a direct reflection of STVA perfusion and only captures contrast medium leakage in the extravascular space. ${ }^{9}$ Conversely, arterial spin-labeling (ASL) is an MR imaging technique that allows quantitative measurement of tissue perfusion by using magnetically labeled blood, therefore without any contrast medium injection. ${ }^{10}$ Accumulating evidence has demonstrated the reliability of ASL in evaluating regional perfusion and its utility in the diagnostic work-up of vascular anomalies. $^{10-13}$ Of particular interest in pediatric patients, waiving the need for contrast injection, ASL is an interesting asset for the diagnosis of STVAs in this population.

The aim of our study was to retrospectively assess the potential value of the ASL sequence for the characterization of cervicofacial soft-tissue vascular anomalies in children.

\section{MATERIALS AND METHODS \\ Study Population}

The study population was derived from a prospective longitudinal cohort of consecutive children referred to Necker Children's Hospital, a tertiary care pediatric hospital, between January 2012 and January 2014. Our STVA registry is a prospectively implemented data base collecting demographic, clinical, imaging, and treatment data of consecutive patients with STVAs admitted at our institution. The initial clinical examination was performed by experienced pediatric dermatologists, and all cases were discussed at a pediatric multidisciplinary meeting, including head and neck surgeons, pediatric radiologists, and interventional neuroradiologists.

This data base was retrospectively queried to identify patients matching the following inclusion criteria: 1) children between birth and 18 years of age; 2) those with cervicofacial STVAs (defined by clinical examination, ultrasound examination, CT angiography, or venography); and 3) patients who had undergone an MR imaging ASL sequence during their initial diagnostic work-up before any treatment or biopsy.

Final diagnosis was obtained prospectively for each patient from the conclusions of our multidisciplinary symposium, which included pediatric dermatologists, radiologists, and surgeons, on the basis of the clinical-imaging features and evolution of the lesion and the pathologic examination findings, when available.

\section{Test Method}

MR Imaging Acquisition. An ASL perfusion sequence was acquired by using a $1.5 \mathrm{~T}$ MR imaging scanner (Signa HDxt; GE Healthcare, Milwaukee, Wisconsin) and consisted of a concurrent spiral 3D pseudocontinuous ASL (12-channel head coil; 3D
Table 1: Population characteristics ${ }^{\mathrm{a}}$

\begin{tabular}{lc}
\hline \multicolumn{1}{c}{ Variable } & Value \\
\hline Age at diagnosis (yr) & $1.45(0.4-5.1)$ \\
Age categories (yr) & \\
0-1 & $17(37 \%)$ \\
1-5 & $18(39.1 \%)$ \\
Older than 5 & $11(23.9 \%)$ \\
Female sex & $27(58.7 \%)$ \\
Localization & \\
Head & $41(89.1 \%)$ \\
Neck & $5(10.9 \%)$ \\
Precise location & \\
Cheek & $5(10.9 \%)$ \\
Submental region & $5(10.9 \%)$ \\
Eyelid & $4(8.7 \%)$ \\
Parotid gland & $4(8.7 \%)$ \\
Scalp & $4(8.7 \%)$ \\
Lip & $3(6.5 \%)$ \\
Multiple & $3(6.5 \%)$ \\
Other & $7(15.2 \%)$ \\
ISSVA subtypes & \\
Hemangioma & $21(45 \%)$ \\
Infantile hemangioma & $18(39.1 \%)$ \\
NICH & $2(4.3 \%)$ \\
RICH & $1(2.2 \%)$ \\
Lymphatic malformation & $10(21.7 \%)$ \\
Common venous malformation & $9(19.6 \%)$ \\
Other (hamartoma, AVF, AVM) & $3(6.7 \%)$ \\
Total No. of patients in study & 46 \\
\hline Note:-1SSVA & \\
\hline
\end{tabular}

Note:-ISSVA indicates International Society for the Study of Vascular Anomalies. ${ }^{a}$ Values are expressed as absolute number (percentage of total) or median (IQR).

volume with a 4-mm section thickness; number of excitations, 3; axial partitions, 80 ; FOV , $240 \times 240$; acquisition matrix, $4 \mathrm{~mm}^{3}$; spiral arms in each 3D partition, 8; points per arm, 512; TE, 10.5 $\mathrm{ms}$; TR, $4428 \mathrm{~ms}$; postlabeling delay, $1025 \mathrm{~ms}$; flip angle, $\left.155^{\circ}\right)$. All patients underwent a routine MR imaging examination, including $\mathrm{T} 1$ and $\mathrm{T} 2$ sequences (including fat saturation when needed); additional sequences were performed at the discretion of the attending radiologist (contrast medium injection, dynamic injection).

MR Imaging Analysis. Regional flow measurements (in milliliters/100 g/minute) were obtained by using an optimized dedicated in-house postprocessing software on Advantage Windows workstations (GE Healthcare), as previously described. ${ }^{14}$ Quantitative measurements were performed in consensus by 2 trained pediatric radiologists (F.B., V.D.-R.), blinded to clinical data. Lesional flow was measured in spheric calibrated ROIs of variable sizes according to the STVA size (mean, $50 \mathrm{~mm}^{2}$ ), positioned in the center of the lesion, with care being taken to avoid partial volume averaging from adjacent tissues.

A third reader (O.N.) performed quantitative measurements independently to allow the calculation of interrater agreement statistics.

\section{Ethics}

The study conformed to generally accepted scientific principles and to ethical standards of research and was approved by an advisory opinion of the Ethics Committee (Comité de Protection des Personnes Ile de France III, AC051) at Necker Children's Hospital. The manuscript was prepared in accordance with Strengthening the Reporting of Observational Studies in Epidemiology 


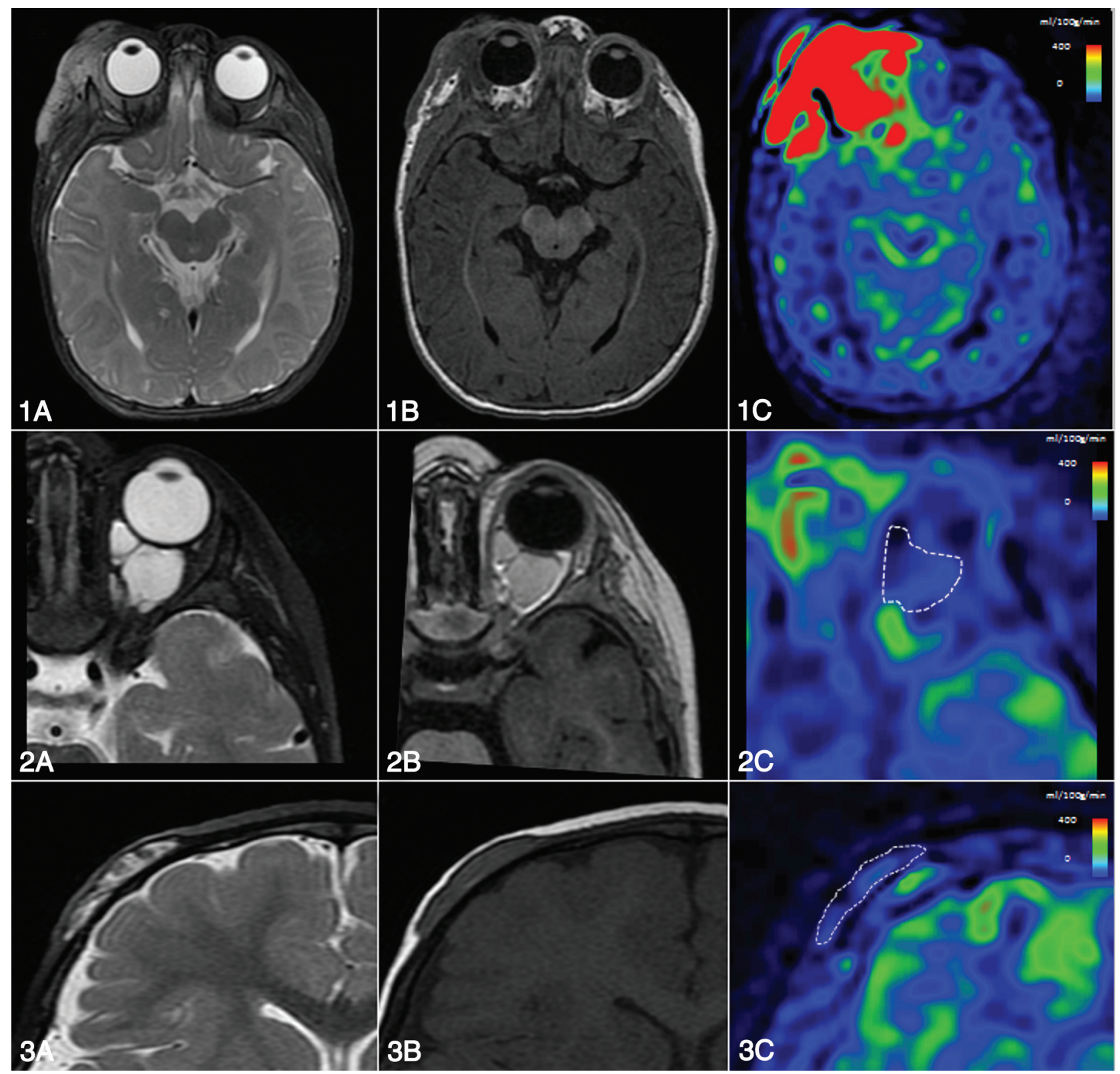

FIG 1. Illustrative cases of blood flow differences in major subtypes of STVA. Blood flow measured with ASL helps distinguish vascular anomalies. A-C, T2, T1, and ASL sequence blood flow maps. 1) Right intra- and periorbital hemangioma in a 6-month-old boy. ASL flow map demonstrates major lesional hyperperfusion (blood flow $=477 \mathrm{~mL} / 100 \mathrm{~g} / \mathrm{min}$ ). 2) Intraorbital venous malformation of a 14-month-old boy with low blood flow $(67 \mathrm{~mL} / \mathrm{min} / 100 \mathrm{~g}) .3)$ Intraosseous cystic lymphangioma of a 13-month-old girl with a low blood flow (45 mL/min/100 g).

guidelines (http://www.equator-network.org/reporting-guidelines/ strobe/).

\section{Statistical Methods}

Values are expressed as mean $\pm \mathrm{SD}$ or median/interquartile range (IQR), as appropriate. For the comparison of flow measurements, the Mood Median test was used due to the unequal variance of samples.

We performed interrater measures by using pair-wise correlations (Pearson product-moment correlation of each pair), comparing mean ASL values of the initial consensus reading with those performed independently by a third reader.

\section{RESULTS}

Among 840 pediatric patients screened for eligibility, 75 in the registry had undergone an ASL sequence at presentation, 49 of whom had a final diagnosis of cervicofacial STVA. Among these 49 patients, the ASL sequence was not interpretable in 3 cases due to kinetic artifacts. The remaining 46 patients were included in the study. Demographic and clinical characteristics of the study population are described in Table 1. The ASL perfusion characteristics in milliliters $/ 100 \mathrm{~g} /$ minute of the 3 most frequent lesional subtypes of STVA are summarized in Fig 1 and Table 2.

Interobserver agreement was excellent for the measurement of ASL perfusion (pair-wise correlation, 0.94; 95\% CI, 0.90-0.97).

\section{Hemangiomas}

Lesion characteristics are detailed in Table 2.

Infantile Hemangioma. For infantile hemangiomas (HIs), the median age was 2.9 months; there were 11 girls and 7 boys. Seven 
Table 2: Summary of baseline clinical and imaging characteristics by diagnostic category in major lesion subtypes ${ }^{2}$

\begin{tabular}{lccc}
\hline \multicolumn{1}{c}{ Variable } & $\begin{array}{c}\text { Hemangiomas } \\
(\boldsymbol{n}=\mathbf{2 1})\end{array}$ & $\begin{array}{c}\text { Common Venous } \\
\text { Malformations }(\boldsymbol{n}=\mathbf{9})\end{array}$ & $\begin{array}{c}\text { Common Lymphatic } \\
\text { Malformations }(\boldsymbol{n}=10)\end{array}$ \\
\hline Age (yr) & $1.46(0.1-8.2)$ & $4.6(1.2-11)$ & $2.65(0.7-11.5)$ \\
Female sex & $12(57)$ & $7(78)$ & $5(50)$ \\
Median ASL flow & $477(212-603)$ & $25(15-66.5)$ & $22.5(16-60)$ \\
T1 signal & Iso: $15(88)$ & Iso: $9(100)$ & Iso: $6(75)$ \\
T2 signal & Hyper: $19(95)$ & Hyper: $9(100)$ & Hyper: $10(100)$ \\
\hline
\end{tabular}

Note:-Iso indicates isointense; Hyper, hyperintense.

${ }^{a}$ Values are expressed as absolute number (percentage of column total) or as mean (range) or median (25th-75th percentiles).

\section{$/ 100 \mathrm{ml} / \mathrm{min}$}

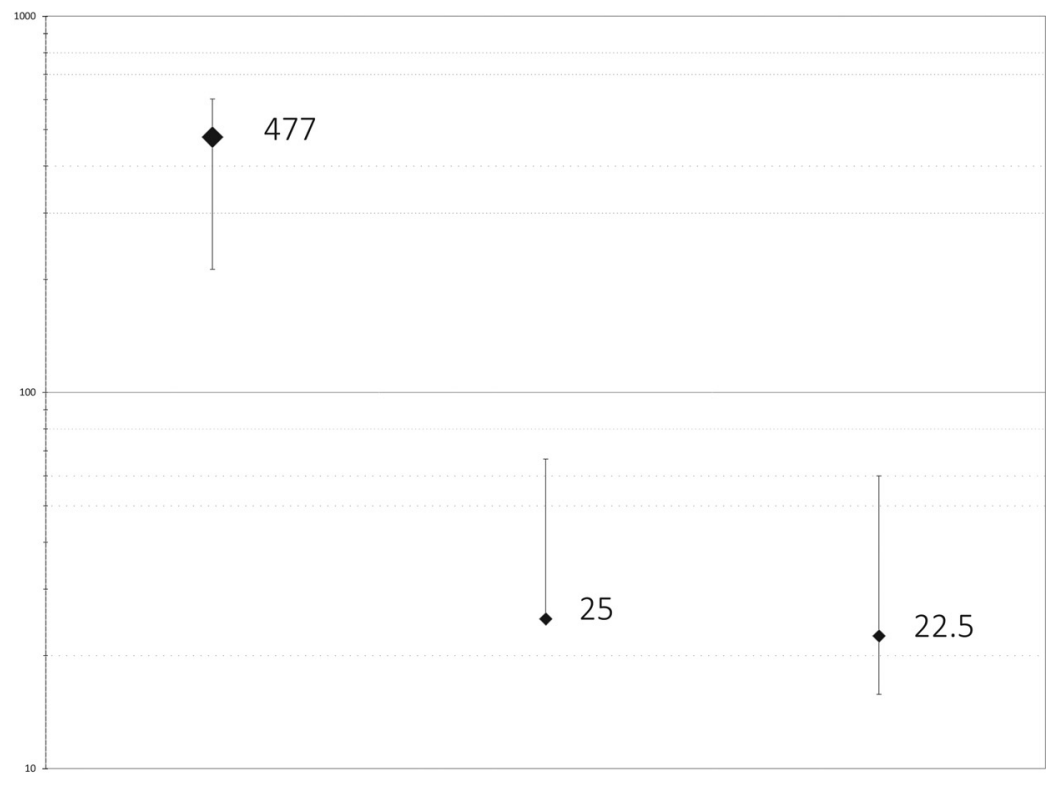

Hemangiomas (IH/RICH/NICH) Common Venous Malformations Common Lymphatic Malformation

\begin{tabular}{|l|l|}
\hline$p<0.001$ & \\
\hline & $p=0.003$ \\
\hline
\end{tabular}

FIG 2. Comparison of major STVA subtypes by using ASL blood flow maps. ASL blood flow values are plotted and labeled median on a 0 - to $1000-\mathrm{mL} / 100 \mathrm{~g} / \mathrm{min}$ base 10 logarithmic scale; error bars represent interquartile range. $\mathrm{IH}$ indicates infantile hemagioma; $\mathrm{RICH}$ and $\mathrm{NICH}$ indicate rapidly and noninvoluting congenital hemangioma.

HIs (39\%) were located in the orbital region. In all cases in which gadolinium injection was performed, the hemangiomas were massively and homogeneously enhanced. All HIs presented with an increased ASL tissue flow. The median perfusion value was 491 $\mathrm{mL} / 100 \mathrm{~g} / \mathrm{min}$ (IQR, $290-622 \mathrm{~mL} / 100 \mathrm{~g} / \mathrm{min}$ ) with a range between 100 and $880 \mathrm{~mL} / 100 \mathrm{~g} / \mathrm{min}$.

Rapidly and Noninvoluting Congenital Hemangioma. Two patients (6.1 and 8.2 years of age, respectively) had noninvoluting congenital hemangioma (NICH), and 1 patient ( 0 years) had rapidly involuting congenital hemangioma ( $\mathrm{RICH})$. After contrast injection, all 3 lesions enhanced massively. They presented with increased ASL-measured flow (median, 196 mL/100 g/min; IQR, $126-424 \mathrm{~mL} / 100 \mathrm{~g} / \mathrm{min})$.

Overall, ASL showed a marked perfusion increase in all hemangiomas (HI, NICH, and RICH), with a median perfusion value of $477 \mathrm{~mL} / 100 \mathrm{~g} / \mathrm{min}$ (IQR, 212.5-603 mL/100 g/min).

\section{Venous Malformations}

For venous malformations, the median age was 4.6 years (range, 1.2-11years). There were 7 girls and 2 boys. All venous malformations presented with typical MR imaging features: isointense on T1 and moderately hyperintense on T2 fatsaturated sequences, with multiple small hypointense nodules representing focal nodular thromboses, precursors of phleboliths (uncalcified or soft phleboliths). The median flow was very low in all lesions (25 mL/min/100 g; IQR, 15-66.5 $\mathrm{mL} / \mathrm{min} / 100 \mathrm{~g})$.

\section{Lymphatic Malformations}

For lymphatic malformations, the median age was 2.65 years (range, 8 months to 11.5 years). There were 5 girls and 5 boys. One patient (10\%) presented with a fluid/fluid level. None of the lesions presented with enhanced perfusion (median flow, $22.5 \mathrm{~mL} / 100 \mathrm{~g} / \mathrm{min}$; IQR, $16-60 \mathrm{~mL} / \mathrm{min} / 100 \mathrm{~g}$ ).

\section{Other Lesion Subtypes}

Both AVMs and AVFs demonstrated increased intralesional perfusion (respectively, 779 and $451 \mathrm{~mL} / 100 \mathrm{~g} / \mathrm{min}$ ), and none of the remaining lesions (namely, 1 hamartoma and 2 capillary lymphatic malformations) showed measurable increased perfusion (data not shown).

\section{Lesion Subtype Comparisons}

Hemangiomas, including $\mathrm{NICH}$ and $\mathrm{RICH}$, presented with significantly higher flows than lymphatic $(P<.0001)$ or venous malformations $(P=.003)$. There was no significant difference between lymphatic and venous malformations (Fig 2).

\section{DISCUSSION}

We demonstrated that the ASL sequence, through its ability to quantitatively measure lesional flow, helps discriminate major subtypes of STVAs. In addition to providing increased lesion conspicuity, the ASL sequence is an excellent tool for refining the diagnosis. In our cohort, ASL was able to characterize an increased perfusion in all hemodynamically active lesions (HI, RICH, NICH, AVM, and AVF) and the absence of any measurable increase of perfusion in the others (lymphatic malformation, venous malformation, and hamartoma) and thus provided decisive help in making the diagnosis. This difference reached statistical significance when we compared the most frequent lesion subtypes (infantile hemangioma, lymphatic malformation, and venous malformation).

These findings provide strong support for the use of the ASL sequence in pediatric patients with STVAs. To date, the role of ASL in the diagnostic work-up of cervicofacial regions has been 
examined in only a handful of studies. ${ }^{15-17}$ Our systematic approach sheds light on the diagnostic possibilities of ASL in pediatric cervicofacial soft-tissue lesions.

Whereas vascular anomalies in children are commonly diagnosed by a combination of clinical examination and ultrasound Doppler findings, CT or MR imaging is often used to solve ambiguous cases or when an extended work-up is needed before treatment (nonexhaustively for osseous, complex, or intracranial lesions). MR imaging, in particular, has emerged as a key complementary tool in this setting. ${ }^{5}$ Several studies have reported the use of dynamic contrast-enhanced sequences to characterize softtissue vascular anomalies, ${ }^{8,18-20}$ emphasizing the paramount importance of the analysis of the hemodynamics of these lesions. ${ }^{21}$ The present exploratory pilot study demonstrates the ability of the ASL sequence to discriminate infantile hemangiomas from venous and lymphatic malformations. Moreover, in view of its ability to quantitatively measure perfusion changes, the ASL sequence also has potential implications for patient follow-up during or after treatment. The blood flow response during or after treatment could easily be assessed by using ASL, but further longitudinal studies will be needed to examine its usefulness in this context.

Caution is needed regarding the interpretation of our results and those of future studies. ASL measures tissue perfusion-that is, the volume of blood that crosses the sample per minute per $100 \mathrm{~g}$ of tissue weight. On the other hand, gadolinium contrast accumulation in the extravascular space and intravascular signal change are responsible for enhancement; hence, the "enhancement" that is seen in structural T1-weighted MR imaging after gadolinium infusion is distinct from tissue perfusion characteristics captured by the ASL sequence. Thus, the terms "hyperperfused" and "enhancing" must not be considered interchangeable. Additionally, the presence of arteriovenous shunts might induce a bias in the perfusion measure because blood is diverted from tissue into the venous side and the diverted labeled blood is not taken into account for the flow measurement. The effect of this technical caveat most certainly accounts for the unexpectedalbeit not significant and derived from a very small number of observations-relatively lower perfusion of AVM in our sample compared with HIs. This limitation could be addressed in the future by using multidelay pseudocontinuous ASL, which allows the characterization of blood pools with variable speeds. ${ }^{22}$

Our study has some limitations. The first is its retrospective design and the associated biases. Also, given the absence of normalized atlas values for soft-tissue lesion flow alteration measurements, we chose to compare the most frequent lesions and could not assess the relative perfusion with adjacent healthy soft tissues. Given the wide variety of STVAs, it was not possible to establish a control group for flow comparison. Finally, even if STVAs were classified in our sample by using a careful and systematic diagnostic approach, our criterion standard for the final diagnosis was based on the conclusions of a multidisciplinary symposium (and not a biopsy in most cases). Thus, we did not compare the diagnostic capacities of ASL with those of conventional noncontrast sequences (T1- and T2-weighted) because the final diagnosis was not made on those sequences alone but on a body of clinicalimaging data. Furthermore, a degree of bias in the classification cannot be ruled out because ASL (qualitatively high or low) may, in some instances, have been used as an additional argument for lesion classification.

\section{CONCLUSIONS}

This study of 46 children with a cervicofacial vascular anomaly demonstrates the potential role of ASL in discriminating major vascular lesion subtypes and assessing the hemodynamics of STVAs. In view of its ability to quantitatively measure lesional flow, ASL could be a useful tool for the follow-up of patients with hemodynamically active STVAs who are under treatment. Further studies will be needed to test this hypothesis.

Disclosures: Olivia Boccara—UNRELATED: Consultancy: Fabre Dermatology; Payment for Lectures Including Service on Speakers Bureaus: Pierre Fabre Dermatology; Travel/Accommodations/Meeting Expenses Unrelated to Activities Listed: Pierre Fabre Dermatology.

\section{REFERENCES}

1. Esterly NB. Kasabach-Merritt syndrome in infants. J Am Acad Dermatol 1983;8:504-13 CrossRef Medline

2. Navarro OM, Laffan EE, Ngan BY. Pediatric soft-tissue tumors and pseudo-tumors: MR imaging features with pathologic correlation, part 1: imaging approach, pseudotumors, vascular lesions, and adipocytic tumors. Radiographics 2009;29:887-906 CrossRef Medline

3. Wassef M, Blei F, Adams D, et al; ISSVA Board and Scientific Committee. Vascular anomalies classification: recommendations from The International Society for the Study of Vascular Anomalies. Pediatrics 2015;136:e203-14 CrossRef Medline

4. Trop I, Dubois J, Guibaud L, et al. Soft-tissue venous malformations in pediatric and young adult patients: diagnosis with Doppler US. Radiology 1999;212:841-45 CrossRef Medline

5. Flors L, Leiva-Salinas C, Maged IM, et al. MR imaging of softtissue vascular malformations: diagnosis, classification, and therapy follow-up. Radiographics 2011;31:1321-40; discussion 1340-41 CrossRef Medline

6. Flors L, Leiva-Salinas C, Norton PT, et al. Ten frequently asked questions about MRI evaluation of soft-tissue vascular anomalies. $\mathrm{Am} J$ Roentgenol 2013;201:W554-62 CrossRef Medline

7. Laffan EE, Ngan BY, Navarro OM. Pediatric soft-tissue tumors and pseudotumors: MR imaging features with pathologic correlation, part 2: tumors of fibroblastic/myofibroblastic, so-called fibrohistiocytic, muscular, lymphomatous, neurogenic, hair matrix, and uncertain origin. Radiographics 2009;29:e36 CrossRef Medline

8. Ohgiya Y, Hashimoto T, Gokan T, et al. Dynamic MRI for distinguishing high-flow from low-flow peripheral vascular malformations. AJR Am J Roentgenol 2005;185:1131-37 CrossRef Medline

9. Sharafuddin MJ, Sundaram M. The role of magnetic resonance imaging in the diagnosis of soft tissue lesions. Crit Rev Diagn Imaging 1994;35:379-483 Medline

10. Petersen ET, Zimine I, Ho YC, et al. Non-invasive measurement of perfusion: a critical review of arterial spin labelling techniques. $\mathrm{Br}$ Radiol 2006;79:688-701 CrossRef Medline

11. Heijtel DF, Mutsaerts HJ, Bakker E, et al. Accuracy and precision of pseudo-continuous arterial spin labeling perfusion during baseline and hypercapnia: a head-to-head comparison with ${ }^{15} \mathrm{O}_{2} \mathrm{O}$ positron emission tomography. Neuroimage 2014;92:182-92 CrossRef Medline

12. Kistner I, Ott C, Jumar A, et al. 1D.09: Applicability of measurement of renal perfusion using 1.5 Tesla MRI arterial spin labelling. $J \mathrm{Hy}$ pertens 2015;33(suppl 1):e17 CrossRef Medline

13. Iv M, Fischbein NJ, Zaharchuk G. Association of developmental venous anomalies with perfusion abnormalities on arterial spinlabeling and bolus perfusion-weighted imaging. J Neuroimaging 2015;25:243-50 CrossRef Medline

14. Blauwblomme T, Naggara O, Brunelle F, et al. Arterial spin labeling magnetic resonance imaging: toward noninvasive diagnosis and 
follow-up of pediatric brain arteriovenous malformations. J Neurosurg Pediatr 2015;15:451-58 CrossRef Medline

15. Pollock JM, Tan H, Kraft RA, et al. Arterial spin-labeled MRI perfusion imaging: clinical applications. Magn Reson Imaging Clin N Am 2009;17:315-38 CrossRef Medline

16. Pollock J, Kraft R, Tan H, et al. Arterial spin labeled perfusion imaging of the orbit: initial experience. In: Proceedings of the Annual Meeting of the American Society of Head and Neck Radiology, Toronto, Ontario, Canada. September 10-14, 2008

17. Mamlouk MD, Hess CP. Arterial spin-labeled perfusion for vascular anomalies in the pediatric head and neck. Clin Imaging 2016;40: 1040-46 CrossRef Medline

18. Thawait SK, Puttgen K, Carrino JA, et al. MR imaging characteristics of soft tissue vascular anomalies in children. Eur J Pediatr 2013;172: 591-600 CrossRef Medline
19. Lidsky ME, Spritzer CE, Shortell CK. The role of dynamic contrastenhanced magnetic resonance imaging in the diagnosis and management of patients with vascular malformations. J Vasc Surg 2012; 56:757-64.e1 CrossRef Medline

20. Masand P. Radiographic findings associated with vascular anomalies. Semin Plast Surg 2014;28:69-78 CrossRef Medline

21. Wang J, Licht DJ, Jahng GH, et al. Pediatric perfusion imaging using pulsed arterial spin labeling. J Magn Reson Imaging 2003;18:404-13 CrossRef Medline

22. Qiu D, Straka M, Zun Z, et al. CBF measurements using multidelay pseudocontinuous and velocity-selective arterial spin labeling in patients with long arterial transit delays: comparison with xenon CT CBF. J Magn Reson Imaging 2012;36:110-19 CrossRef Medline 